MASA M X

\title{
POLARIZATION AND INTENSITY MEASUREMENTS OF HYDROGEN LYMAN ALPHA IN THE DAY AIRGLOW
}

\author{
BY \\ DONALD F. HEATH
}

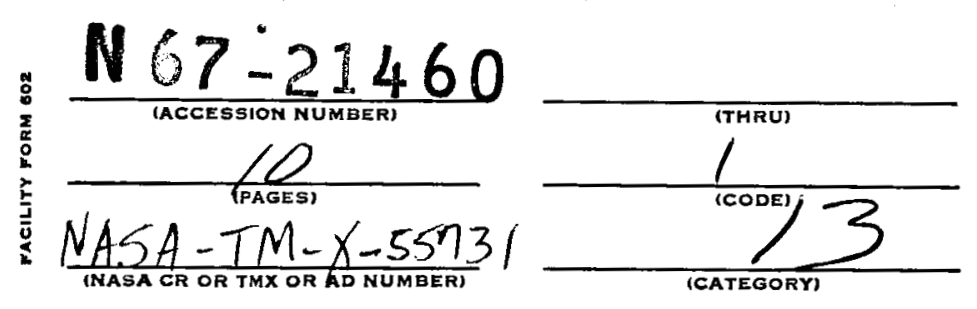

MARCH 1967 
POLARIZATION AND INTENSITY MEASUREMENTS OF HYDROGEN LYMAN ALPHA IN THE DAY AIRGLOW

by

Donald F. Heath

March 1967

Goddard Space Flight Center

Greenbelt, Maryland 


\section{POLARIZATION AND INTENSITY MEASUREMENTS OF HYDROGEN LYMAN ALPHA IN THE DAY AIRGLOW}

Since the discovery of hydrogen Lyman alpha in the night airglow in 1955 by Byram, et al., (1957) and the spectrophotometric measurements of the night and day airglow in 1962 by Fastie, et al., (1964) numerous experiments have been flown and theories proposed to explain the source, and the intensity of $\mathrm{Ly}-\alpha^{1}$. Brandt and Chamberlain (1960) discussed the importance of a polarization measurement for understanding the hydrogen problem.

This paper is concerned with the measurements of polarization and intensity in the day airglow of $\mathrm{Ly}-\alpha$. The polarization and intensity were measured as a function of altitude for the principal far ultraviolet day airglow emission features from $1200 \mathrm{~A}$ to $2000 \mathrm{~A}$. These included the hydrogen $\mathrm{Ly}-\alpha$ line at $1216 \mathrm{~A}$, the resonance lines of $\mathrm{OI}$ at $1304 \mathrm{~A}$, and the group at $1355 \mathrm{~A}$, and an unidentified source of emission in the region from $1450 \mathrm{~A}$ to $2000 \mathrm{~A}$. The experimental details of the multichannel, far ultraviolet, polarization photometer, and the measurements of the features other than Ly- $\alpha$ will be treated in separate papers.

This experiment, with experiments for measuring direct solar Ly-a, the broad band solar flux in four intervals from $1450 \mathrm{~A}$ to $3000 \mathrm{~A}$ and two electron density and temperature probes were flown on an Aerobee 150 rocket from White Sands, New Mexico. The flight from WSMR (latitude $32^{\circ} 24^{\prime}$ north, longitude

\footnotetext{
${ }^{1}$ A summary of these works is contained in a review paper by Donahue (1966)
} 
$7^{\mathrm{h}} 05^{\mathrm{m}}$ west) was initiated at 8:20 A.M. M.S.T. on August 29, 1966. At the time of launching, the three hour geomagnetic planetary index was $\mathrm{Kp}=2$. During the previous 3 hour period, the index was $\mathrm{Kp}=4$, which marked the end of 5 quiet days. The rocket carried, in addition to its experimental payload, a solar aspect sensor and two magnetometers. The attitude of the rocket was controlled by an inertial attitude control system ${ }^{2}$, and it reached a peak altitude of $200 \mathrm{~km}$. At the time of launch the solar zenith angle was $58^{\circ}$ and its azimuth $10^{\circ}$ south of east. The geomagnetic field at WSMR is at $60^{\circ}$ elevation, and $12.5^{\circ}$ west of south in azimuth. A zenith-azimuth angle plot of the photometer view as a function of flight time is given in figure 1.

The polarization photometer had a field of view of $4.0^{\circ}\left(0.39 \times 10^{-2}\right.$ steradians $)$. A geneva action filter wheel successively interposed; no filter, calcium fluoride, barium fluoride, sapphire, and a blank ${ }^{3}$. For each filter position the analyzer rotated through an angle of $720^{\circ}$. The filters provided the short wavelength cutoffs, and the "solar blindness" of the cathode of an ASCOP 542G photomultiplier limited the long wavelength response. Consequently the signals from the five filter wheel positions were due to radiation from $1200 \mathrm{~A}-2000 \mathrm{~A}, 1230 \mathrm{~A}-2000 \mathrm{~A}, 1335 \mathrm{~A}-$ 2000A, 1435A-2000A, and the dark current. The signal currents were detected and converted into a telemetry input voltage with a four decade range switching linear electrometer. ${ }^{4}$ Subtractive techniques were used to isolate the signal in wavelength intervals $1435 \mathrm{~A}-2000 \mathrm{~A}, 1335 \mathrm{~A}-1435 \mathrm{~A}, 1230 \mathrm{~A}-1335 \mathrm{~A}$, and $1200 \mathrm{~A}-$ $1230 \mathrm{~A}$. The relative intensities are accurate to $\pm 2-3 \%$, while the absolute intensities which were obtained by calibration against a NO ionization cell are thought to

\footnotetext{
2 Aerojet General Corp.

3 The filter wheel photometer was fabricated by the Ray Lee Machine Co.

4 Adcole Corp.
} 
be accurate to $\pm 15 \%$. In reducing the data it was assumed that the principal day airglow emission in the interval from $1200 \mathrm{~A}-1435 \mathrm{~A}$ are those reported by Fastie, et al. 1964.

The transmission polarization analyzer consisted of 8 cleaved plates of lithium fluoride which were set at Brewster's angle for the incident Ly- $a$ radiation..$^{5}$ The analyzer had measured major and minor principal transmittances at $1216 \mathrm{~A}$ of $\mathrm{k}_{1}=0.088$ and $\mathrm{k}_{2}=0.0037$ respectively. The transmittance of the analyzer for unpolarized light is given by the relation.

$$
\mathrm{T}=\frac{1}{2}\left(\mathrm{k}_{1}+\mathrm{k}_{2}\right)
$$

where $\mathrm{k}_{1}$ and $\mathrm{k}_{2}$ are transmittances measured parallel to and perpendicular to the plane of maximum transmission for linear polarized light. The degree of linear polarization is given by the relation

$$
P=\frac{k_{1}+k_{2}}{k_{1}-k_{2}}\left(\frac{I_{\text {max }}-I_{\text {min }}}{I_{\text {max }}+I_{\text {min }}}\right)
$$

where $I_{\max }$ and $I_{\min }$ are the maximum and minimum of the telemetry signals resulting from the rotation of the analyzer.

The altitude profile of the intensity of Ly-a (kilorayleighs) for the down leg portion of the flight is given in figure 2.6 Prior to $t=234$ seconds all radiation channels showed evidence of scattered light. This corresponds to solar illumination of the photometer axis at angles less than $80^{\circ}$. The maximum intensity

\footnotetext{
5 The polarization analyzer was fabricated by the Harshaw Chemical Co.

${ }^{6}$ For $L y-a 1 k R=1.30 \times 10^{-3} \mathrm{ergs} / \mathrm{cm}^{2} \mathrm{sec}$ steradian
} 
was about $20 \mathrm{kR}$ with a possible increase of $1 \mathrm{kR}$ between $200 \mathrm{~km}$ and $130 \mathrm{~km}$. The triangular and the adjacent circular data points give the magnitude of the horizon-azimuth effect which amounts to an increase of $37 \%$ in going from a zenith angle of $32^{\circ}$ to $65^{\circ}$ and in azimuth from $304^{\circ}$ to $351^{\circ}$.

The direct solar Ly-a flux measured at apogee was $5.8 \mathrm{ergs} / \mathrm{cm}^{2} \mathrm{sec}$.

No polarization of Ly-a was detectable, and from an analysis of the telemetry records one can state that the maximum possible polarization is less than 1.5\%. Two sample telemetry records are shown in figure 3. The upper one (A) is representative of the $\mathrm{Ly}-\alpha$ data after $t=234$ sec. The lower record (B) is typical of those showing the effect of sunlight which was polarized $(P=19 \%)$ by scattering off the baffle of the photometer. For (B) the solar flux was incident at $58^{\circ}$ to the axis of the photometer.

The polarization of resonance radiation is directly related to the Zeeman effect which depends upon level hyperfine structure. Brandt and Chamberlain (1960) have calculated that at $90^{\circ}$ to the sun the total $\mathrm{Ly}-\alpha$ line should show a polarization of $16 \%$ for completely separated hyperfine levels, and $27 \%$ for no hyperfine splitting of the upper ${ }^{2} \mathrm{P}_{3 / 2}$ level. These results are for single scattering so consequently the polarization would be reduced for multiple scattering.

Donahue (1966) made resonance scattering calculations of the zenith intensity above $120 \mathrm{~km}$, as a function solar zenith angle for different optical thicknesses measured above $100 \mathrm{~km}$ based on the 1962 solar flux. A zenith intensity of 14 $\mathrm{kR}$ for a solar zenith angle of $60^{\circ}$ would require about 3.5 optical thickness above $100 \mathrm{~km}$, or about 1.8 optical thicknesses above $120 \mathrm{~km}$. The problem which 
should be investigated is how many optical thicknesses of hydrogen are required above $200 \mathrm{~km}$ so that an initial polarization of $16-27 \%$ for single resonance scattering is reduced to less than $1.5 \%$ through multiple scattering.

Alternatively Kondo and Kupperian (1967) have proposed that a part of the diffuse night $\mathrm{Ly}-\alpha$ glow is due to the interaction of neutral hydrogen with the charged particles in the radiation belts. Their theory predicts a general increase of intensity in the direction of the geomagnetic poles, and a decrease in the direction of the geomagnetic equator. This mechanism does offer a source of unpolarized radiation.

In conclusion, the polarization photometer measurements require a composite source to explain the Ly-a day airglow. Multiple resonance scattering is required to explain the absence of polarization. In support of this there are the recent mass spectrometer measurements on Explorer 32 by Reber (1967) which indicate there is much more neutral hydrogen than was previously believed. Finally a collisional source of excitation such as proposed by Kondo and Kupperian (1967) is necessary to explain the horizon brightening.

\section{REFERENCES}

Fastie, W. G., Crosswhite, H. M., and Heath, D. F.1964, J. Geophys. Res., $\underline{69}, 4129$. Byram, E. T., Chubb, T. A., Friedman, H. and Kupperian, J. E. 1957, "Threshold of Space," ed. M. Zelikoff (New York, Pergamon Press), p. 203.

Donahue, T. M. 1966, Ann. Geophys. 22, 175.

Brandt, J. C., Chamberlain, J. W. 1960, Ap. J., 130, 670.

Kondo, Y., Kupperian, J. E., Jr. 1967, Submitted to J. Geophys. Res.

Reber, C. A. 1967, private communication. 


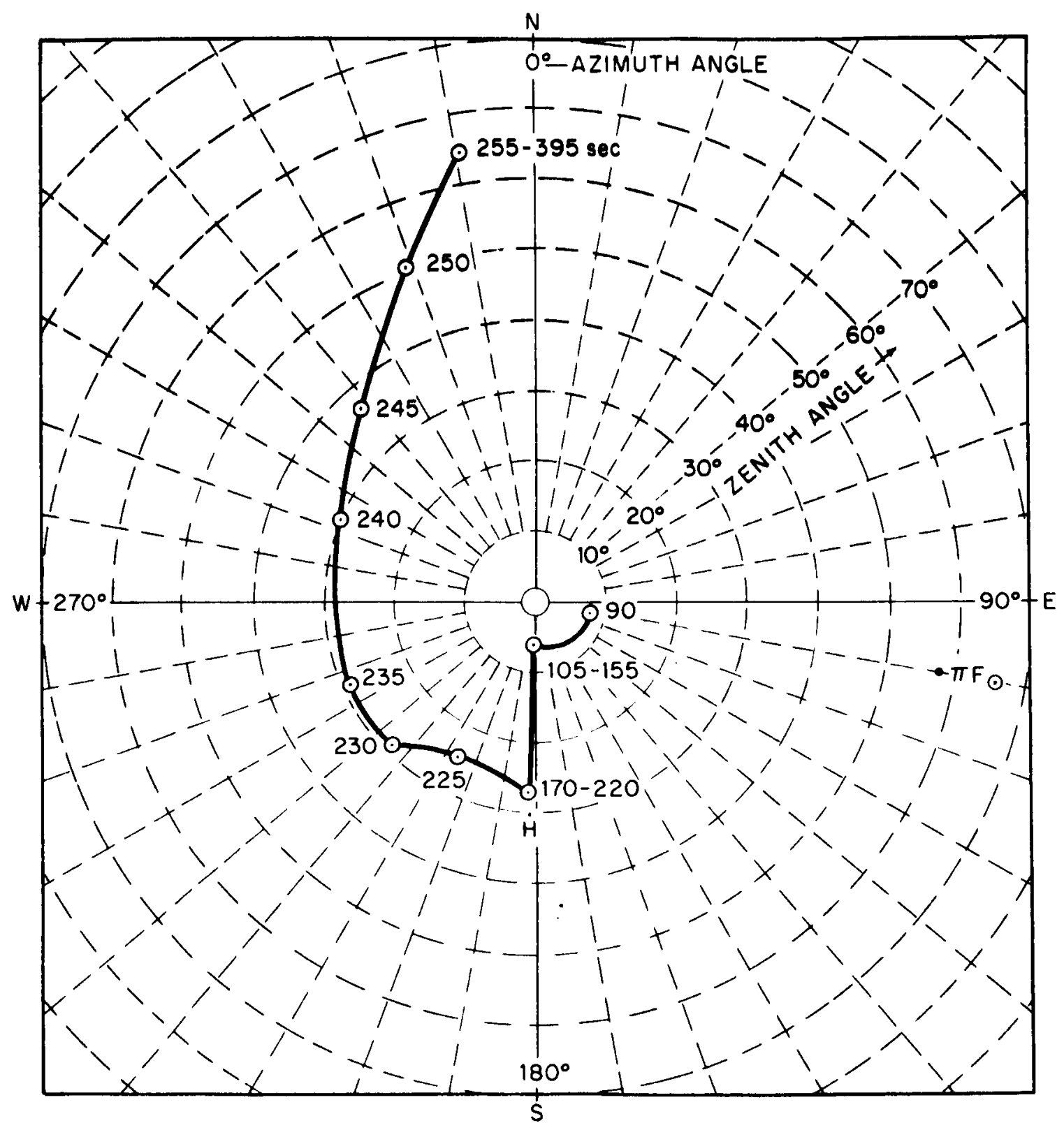

Figure 1-Variation of zenith and azimuth angles of the photometer axis with time. 


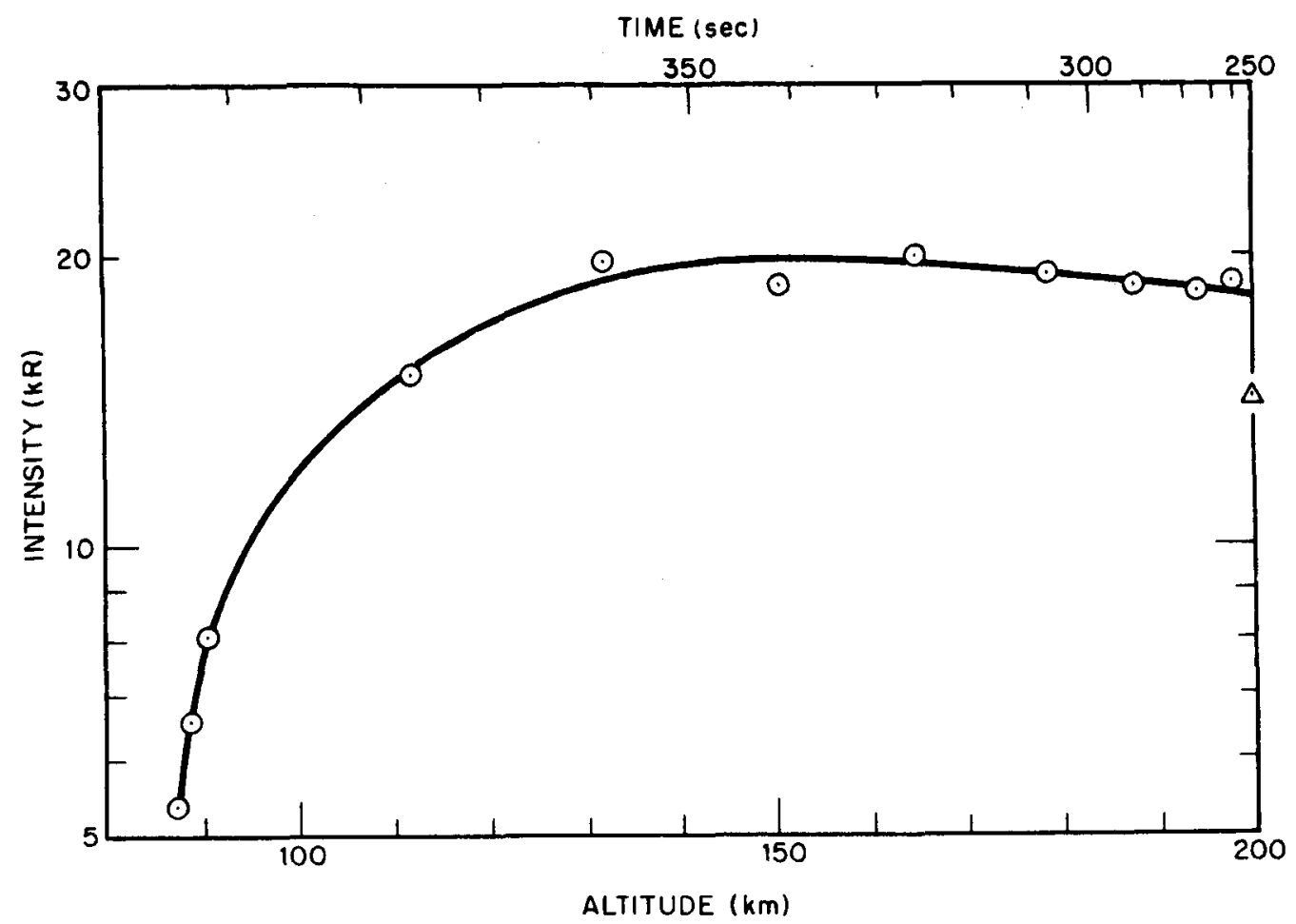

Figure 2-Lyman alpha altitude profile for downward leg of flight. All data points indicated by circles for zenith angle $65^{\circ}$ and azimuth $9^{\circ}$ west of north. The photometer axis was at $92.5^{\circ}$ from the sun and $90.5^{\circ}$ from the geomagnetic field. The $\Delta$ data point was for a zenith of $32^{\circ}$ and azimuth of $56^{\circ}$ west of north. 

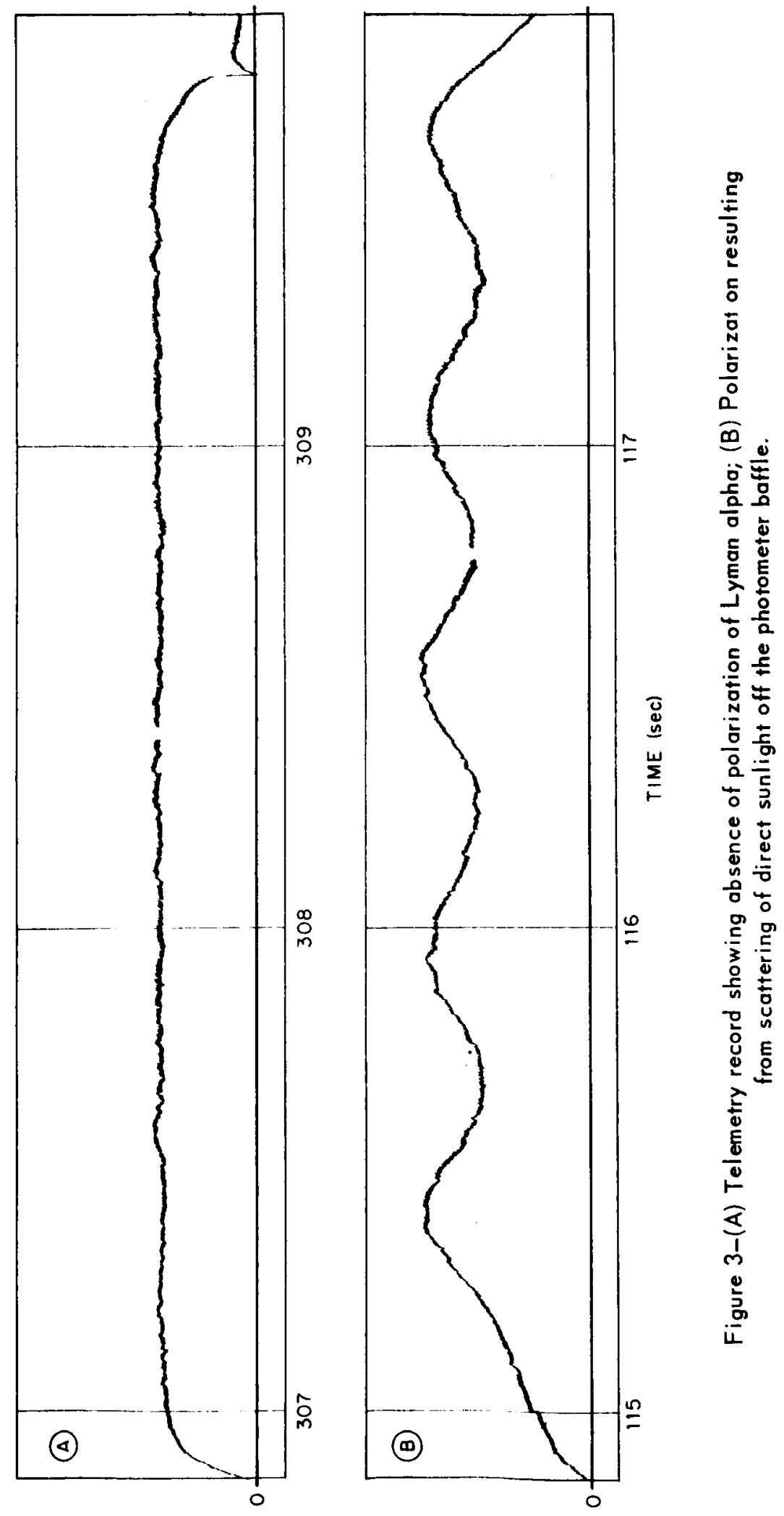